\title{
PRIMER CONTEXTO MORTUORIO DEL HOLOCENO MEDIO EN LA COSTA NORTE DE SANTA CRUZ (PATAGONIA ARGENTINA)
}

LEANDRO ZILIO

\section{RESUMEN}

En esta comunicación se da a conocer el hallazgo de un contexto mortuorio denominado Punta Buque 3, ubicado en la localidad Punta Medanosa, provincia de Santa Cruz, Argentina. El sitio es un entierro en duna de un individuo adulto masculino. Una datación radiocarbónica directa lo sitúa cronológicamente en el Holoceno medio, por lo que representa el primer contexto de entierro datado para este momento en la costa norte de Santa Cruz. Se presentan las determinaciones de los estudios bioantropológicos, tafonómicos, geoquímicos de sedimentos y de isótopos estables de $\delta^{13} \mathrm{C}$ y $\delta^{15} \mathrm{~N}$. Esta información permitió inferir que el sitio corresponde a un entierro de un individuo quien hizo uso complementario de los ambientes costeros y del interior durante el Holoceno medio.

PALABRAS CLAVE: entierro humano, Holoceno medio, cazadores-recolectores, isótopos estables, costa norte de Santa Cruz.

\section{FIRST MORTUARY CONTEXT FROM THE MIDDLE HOLOCENE ON THE NORTHERN COAST OF SANTA CRUZ (PATAGONIA ARGENTINA)}

\begin{abstract}
In this communication a mortuary context denominated as Punta Buque 3, located in the locality of Punta Medanosa on Santa Cruz province, Argentina, is presented. The site is a burial of a male adult individual located within a dune. A direct radiocarbon date places the burial chronologically in the middle Holocene, which represents the first burial dated for this moment on the northern coast of Santa Cruz. Bioanthropological determinations, taphonomic studies, geochemical analysis of sediments and stable isotope of $\delta^{13} \mathrm{C}$ and $\delta^{15} \mathrm{~N}$ are presented. This information allows us to infer that the site corresponds to a burial of an individual who made a complementary use of coastal and inland environments during the middle Holocene.
\end{abstract}

KEY WORDS: human burial, Middle Holocene, hunter-gatherers, stable isotopes, northern coast

a CONICET - División Arqueología, Facultad de Ciencias Naturales y Museo, Universidad Nacional de La Plata. Museo de La Plata, Paseo del Bosque s/nº (1900), La Plata, Argentina. leandrozilio@yahoo.com.ar 
of Santa Cruz.

INTRODUCCIÓN

En esta nota se exponen los resultados de los estudios del entierro Punta Buque 3 (PB3). Este sitio constituye el primer contexto de entierro humano datado en el Holoceno medio para el área de la costa norte de Santa Cruz, Argentina. El sitio PB3 se encuentra en el sector norte de la localidad arqueológica Punta Medanosa (Fig. 1), sobre una duna, cercano a la línea de costa actual, sin ninguna estructura de rocas $\mathrm{u}$ otros materiales asociados. El hallazgo de este entierro se produjo durante tareas de prospección en el campo a partir de las cuales se identificó diversos restos óseos expuestos en superficie debido a los efectos de la deflación eólica (Fig. 2). Asociado a este entierro, aunque no de manera directa, se encuentra un sitio conchero (Fig. 2). Este último se caracteriza particularmente por la presencia de restos de arqueomalacológicos (Hammond, 2013).

Se presentan las determinaciones bioarqueológicas, un fechado radiocarbónico directo, las tendencias paleodietarias inferidas a partir del análisis de isótopos estables de $\delta^{13} \mathrm{C}$ y $\delta^{15} \mathrm{~N}$, el análisis geoquímico de los sedimentos asociados, así como los análisis de las modificaciones tafonómicas de naturaleza no antrópica.

\section{METODOLOGÍA}

Durante las tareas de campo se realizó

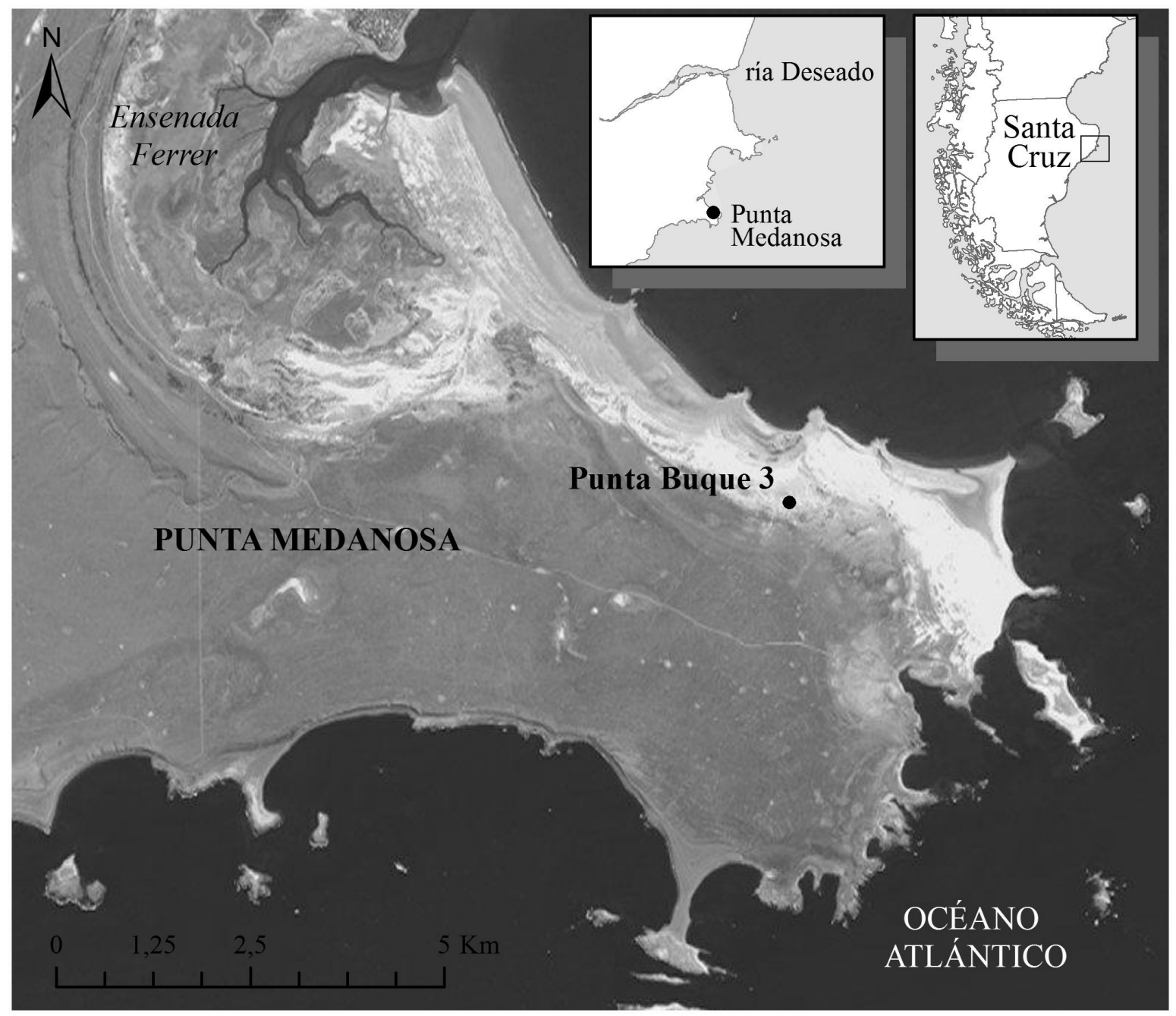

Fig. 1. Mapa de la ubicación del entierro Punta Buque 3. 
un relevamiento detallado de la ubicación y la disposición de las partes esqueletales del individuo hallado. Se demarcó un sector de 1 por $1 \mathrm{~m}$ en el cual se distribuían los restos óseos y el sedimento eólico de tamaño arena que conformaba la duna. En dicho espacio se tamizó con una malla de $2 \mathrm{~mm}$ de apertura. Este tipo de sedimento que se encontraba suelto, sin signos de compactación, permitió la fácil excavación y recuperación de los restos.

La determinación del sexo se realizó a partir del análisis de diversos rasgos morfológicos de la pelvis (Buikstra \& Ubelaker, 1994). La edad de muerte se obtuvo a partir de los estudios de la morfología de la sínfisis púbica (Brooks \& Suchey, 1990), y de la superficie auricular y retroauricular del ilion (Meindl \& Lovejoy, 1989).

Se analizaron distintas variables tafonómicas a fin de evaluar las condiciones de preservación de los restos óseos humanos. Las variables consideradas fueron: la presencia de dióxido de manganeso y carbonato cálcico, marcas de raíces, roedores y carnívoros, los estadios meteorización y el estado de fragmentación de los elementos óseos (Behrensmeyer, 1978; Buikstra \& Ubelaker, 1994; Lyman, 1994; Martin, 2013).

El análisis radiocarbónico se realizó en el Laboratorio de Radiocarbono (CIG-LATYR). El fechado se calibró mediante el software OxCal 4.2 (Bronk Ramsey \& Lee, 2013). La calibración se realizó en años AP, con dos desvíos estándar, empleando la curva de calibración para el Hemisferio Sur (SHCal13) de Hogg et al. (2013).

Se llevaron a cabo análisis de isótopos estables de $\delta^{13} \mathrm{C} y \delta^{15} \mathrm{~N}$ basados en el procesamiento de la fracción orgánica (colágeno) en restos óseos. Las mediciones isotópicas se efectuaron en el Instituto de Geocronología y Geología Isotópica (INGEIS). Se tuvo en cuenta la relación $\mathrm{C} / \mathrm{N}$ con el objetivo de evaluar posibles alteraciones de naturaleza diagenética.

El estudio del sedimento asociado al entierro se realizó en el IGS-CISAUA (Instituto de Geomorfología y Suelos-Centro de Investigaciones en Suelos y Agua de uso agropecuario, perteneciente a la Universidad Nacional de La Plata). Las variables analizadas fueron contenido porcentual de materia orgánica

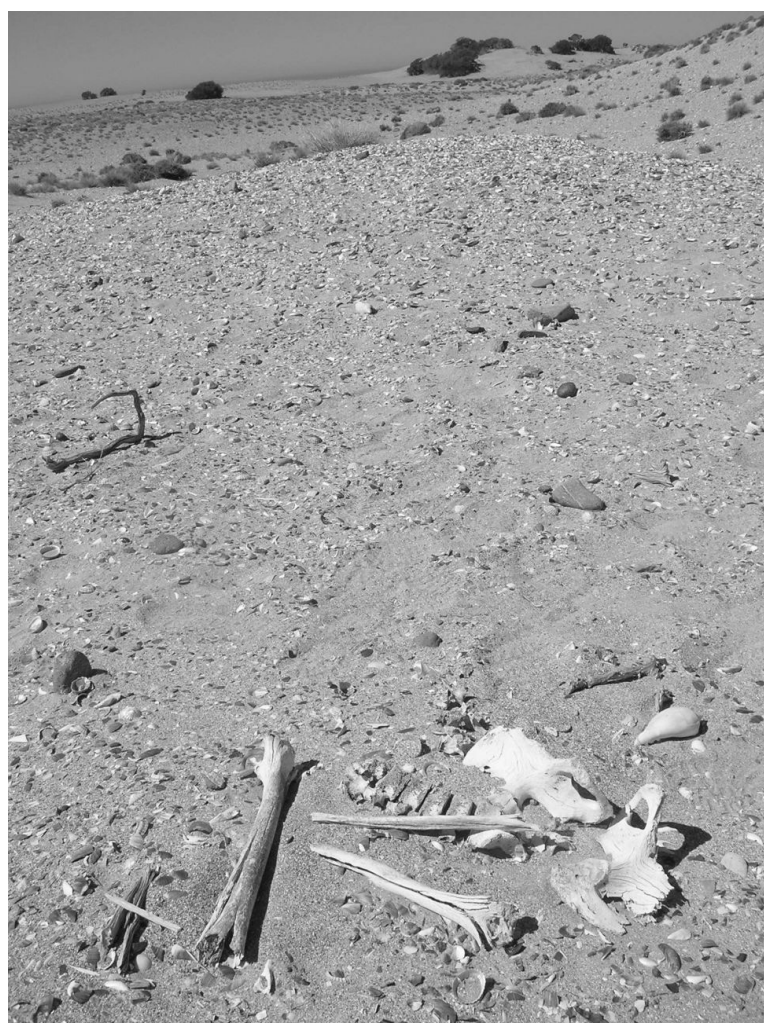

Fig. 2. Fotografía del entierro Punta Buque 3.

carbono orgánico y nivel de $\mathrm{pH}$. RESULTADOS

El sitio presenta signos de alteración por saqueo, evidenciada por la ausencia de diversas piezas esqueletales, como por ejemplo, el cráneo. También se observó que el contexto se encontraba disturbado debido a la movilización de algunas partes esqueletales por la acción del paso de pingüinos de Magallanes (Spheniscus magellanicus) que conforman una gran colonia en la localidad Punta Medanosa (Schiavini et al. 2005).

El entierro está conformado por los siguientes elementos esqueletales: coxal derecho e izquierdo, sacro, omóplato derecho, cuatro fragmentos de vértebras cervicales, una vértebra dorsal, cuatro vértebras lumbares, tres fragmentos de costillas, una epífisis proximal de cúbito derecho, un fragmento diáfisis de peroné izquierdo $y$ de fémur izquierdo y un fragmento de diáfisis y epífisis distal de húmero izquierdo (Fig. 3). El número de elementos representados en el sitio es de 20, los cuales en todos los casos corresponden 
a un individuo $(\mathrm{NMI}=1)$. La mayor parte de ellos presenta fracturas $(\mathrm{n}=14 ; 70 \%)$.

Parte de los restos óseos se encontraban en posición anatómica (vértebras lumbares, sacro y pelvis; Fig. 2), lo cual nos permite inferir que se trata probablemente de un entierro primario en posición decúbito dorsal extendido, aunque se desconoce cómo se encontraban dispuestas sus extremidades y cráneo. A partir de los análisis bioantropológicos realizados sobre los huesos de la pelvis fue posible determinar que se trata de un individuo de sexo masculino. Esto se pudo establecer a partir del análisis de la morfología de la amplitud de la escotadura ciática mayor, del arco ventral, concavidad subpúbica y del borde de la rama isquiopúbica (Buikstra \& Ubelaker, 1994). El estudio de la superficie auricular y retroauricular del ilion (Meindl \& Lovejoy, 1989) permitió determinar que se trataba de un adulto joven de entre 20 y 34 años de edad. Sin embargo, otros indicadores como por ejemplo cambios morfológicos de la sínfisis púbica, no pudieron observarse debido al alto grado de meteorización, o por su ausencia (ej. cráneo).

En conjunto, el material óseo presentó estadios de meteorización dispares. La mayor parte de los elementos se concentraron en los grados 1-3 $(n=13 ; 65 \%)$, aunque se registraron elementos con meteorización grado $4(n=6 ; 30 \%)$ y $1(n=1 ; 5 \%)$. Los elementos óseos en donde se identificaron los estadios de meteorización 1-3, indican la preservación diferencial de las partes óseas, debido a que una parte del hueso quedó expuesta (estadio 3), mientras que el resto permaneció enterrada (estadio 1). En casi la totalidad de los elementos analizados se observaron marcas de raíces $(n=19$; $95 \%$ ). Estas marcas son superficiales por lo que no provocaron modificaciones importantes. No se identificó en ninguno de los huesos la presencia de tinción con óxido de manganeso, carbonato de calcio, marcas de roedores ni de carnívoros. Tampoco se observaron marcas de corte. Sin embargo, es posible que las marcas de radículas, así como la meteorización registrada pudieran enmascarar la observación de otras variables tafonómicas y/o culturales.

Se realizó una datación por ${ }^{14} \mathrm{C}$ sobre un elemento óseo, obteniéndose una edad de 4.970 \pm 100 años AP (LP-3.034). La edad calibrada arrojó un rango de edad de 5.715-5.600 años
AP (mediana=5.639 años AP).

En cuanto a los análisis isotópicos realizados sobre una costilla (AIE 35309), se obtuvieron valores de $\delta^{13} \mathrm{C}$ de $-17,09$ $\%$ y de $\delta^{15} \mathrm{~N}$ de 14,83 \%o, a partir de los cuales se infiere una dieta mixta representada por la ingesta de recursos marinos $y$ terrestres en proporciones relativamente similares (Moreno et al. 2011; Zilio et al. 2014; Zilio, 2015). Estos valores son semejantes a otros obtenidos en restos óseos humanos datados en el Holoceno tardío en el área de la costa norte de Santa Cruz (Zilio, 2015). En la muestra estudiada la relación $\mathrm{C} / \mathrm{N}$, medida sobre el colágeno, es de 3,2. Este valor se ubica dentro del rango aceptado para muestras sin alteración diagenética (De Niro, 1985).

$$
\text { Por debajo de los }
$$

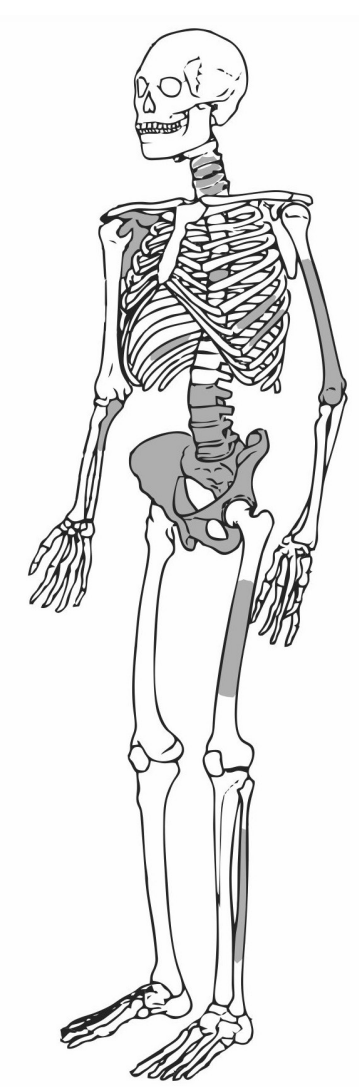

Fig. 3. En color gris representación de elementos óseos del entierro Punta

Buque 3. restos en posición anatómica, se identificó un sedimento arenoso de coloración rojiza y más consolidado que la arena que conformaba la duna. Los resultados de los estudios geoquímicos realizados sobre muestras de este sedimento determinaron un $\mathrm{pH}$ ligeramente alcalino $(8,2)$, con poco contenido de carbono orgánico $(0,09 \%)$ y materia orgánica $(0,16 \%)$. Este entierro se encontraba asociado, aunque no de manera directa, a exoesqueletos calcáreos de moluscos que conforman un sitio conchero. Se interpreta que el valor de $\mathrm{pH}$ podría estar influenciado por la abundancia de carbonato de calcio que conforma la estructura calcárea de las valvas. En general, la abundancia de carbonato de calcio que conforma los exoesqueletos de moluscos genera la formación de un $\mathrm{pH}$ neutro o medianamente alcalino, asociado en este caso 
a matrices constituidas principalmente por arena fina, sustratos salobres cercanos al litoral marítimo y clima árido. Los ambientes alcalinos permiten la preservación de distintos materiales orgánicos, como por ejemplo restos óseos (Claassen, 1998; Orquera \& Piana, 2000).

Se infiere que el individuo recuperado en PB3, podría haber sido envuelto con algún material (por ejemplo cuero o fibras) en el que se introdujo el pigmento rojo y luego enterrado. En la duna, tanto el material envolvente como los tejidos humanos se habrían degradado generando la tinción del sedimento. En los restos óseos no se identificó la coloración rojiza, aunque es probable que no se preservara debido al elevado estado de meteorización de los elementos. Una interpretación similar fue planteada por Gómez Otero y Dahinten (1997-1998) para el entierro La Azucena 1 en la costa de la provincia de Chubut.

\section{DISCUSIÓN Y CONCLUSIONES}

El entierro PB3 constituye el primer contexto mortuorio datado en el Holoceno medio, para la costa norte de Santa Cruz. Este sitio se encuentra aproximadamente a $620 \mathrm{~m}$ de la línea actual de alta marea y a $6 \mathrm{msnm}$, lo que permite inferir que en este sector de costa el nivel del mar no habría variado considerablemente durante el Holoceno tardío.

La presencia de marcas de raíces en los restos óseos analizados puede interpretarse como evidencia de la ocurrencia de procesos edáficos y de la presencia de vegetación en la geoforma en la que se emplazó el entierro. Estos procesos edáficos, aunque sea incipientes, nos señalan momentos de estabilidad y desarrollo de cubierta vegetal.

Respecto de la información paleodietaria, los valores de $\delta^{13} \mathrm{C}$ y $\delta^{15} \mathrm{~N}$ indican una dieta mixta, lo que sugiere un consumo y explotación de recursos marinos y terrestres. Aunque sólo se trata de un caso, las señales isotópicas de PB3 estarían respaldando la hipótesis de la existencia de movimientos individuales entre la costa y el interior, durante el último período del Holoceno medio (Zilio, 2015).

Los restos óseos humanos de este entierro se encontraban expuestos en la duna y cercanos a otros materiales arqueológicos que conforman un sitio conchero (principalmente exoesqueletos calcáreos de moluscos). Sin embargo, no fue posible determinar la causa de la relación espacial entre el contexto mortuorio y el conchero. Se plantean dos posibles explicaciones; por un lado, ambos contextos arqueológicos podrían ser contemporáneos, existiendo una asociación cultural directa. Por el otro, cada registro podría corresponder a diferentes eventos cronológicos, aunque debido a la deflación de la duna en donde se emplazan, se habría generado una mezcla de materiales no contemporáneos o palimpsesto. En Punta Medanosa se ha registrado un fuerte proceso de deflación de los mantos eólicos (Hammond et al. 2013), por lo que la última explicación se considera relevante. Este problema no ha podido ser resuelto hasta el momento, por lo que constituye un interrogante a resolver en la agenda futura.

\section{BIBLIOGRAFÍA}

Behrensmeye, A. (1978). Taphonomic and ecologic information from bone weathering. Paleobiology, 4(2), 150-162.

Bronk Ramsey, C., \& Lee, S. (2013). Recent and planned developments of the program OxCal. Radiocarbon, $55,3-4$.

Brooks, S., \& Suchey, J. (1990 ). Skeletal age determination based on the os pubis: a comparison of the AcsádiNemeskéri and Suchey-Brooks methods. 5(3), 227238.

Buikstra, J., \& Ubelaker, D. (1994). Standards for data collection from human skeletal remains. Arkansas: Archaeological Survey Research Series 44.

Claassen, C. (1998). Shells. Cambridge: University Press.

De Niro, M. (1985). Postmortem preservation and alteration of in vivo bone collagen isotope ratios in relation to paleodietary reconstruction. Nature, 317, 806-809.

Gómez Otero, J., \& Dahinten, S. (1997-1998). Costumbres funerarias $y$ esqueletos humanos: variabilidad $y$ poblamiento en la costa nordeste de la provincia del Chubut (Patagonia Argentina). Relaciones, XXII-XXII, 101-124.

Hammond, H. (2013). Propuesta metodológica para el estudio de conjuntos malacológicos de sitios concheros: su aplicación en la costa norte de Santa Cruz (Patagonia Argentina). Zaranda de Ideas, 9(2), 77-102.

Hammond, H., Zubimendi, M., \& Zilio, L. (2013). Composición de concheros concheros y uso del espacio: aproximación al paisaje arqueológico costero en punta Medanosa. 
Anuario de Arqueología, 5, 67-84.

Hogg, A., Hua, Q., Blackwell, P., Niu, M., Buck, C.,... Guilderson, T. (2013). SHCal13 Southern Hemisphere calibration, 0-50,000 cal yr BP. Radiocarbon, 55(4), 1889-1903

Lyman, R. (1994). Quantitative units and terminology in zooarchaeology. American Antiquity, 59, 36-71.

Martin, F. (2013). Tafonomía de la Transición PleistocenoHoloceno en Fuego-Patagonia. Interacción entre humanos y carnívoros y su importancia como agentes en la formación del registro fósil. Punta Arenas: Ediciones de la Universidad de Magallanes.

Meindl, R., \& Lovejoy, C. (1989). Age changes in the pelvis: implications for paleodemography. En Age markers in the human skeleton (pp. 137-168).

Moreno, E., Zangrando, A., Tessone, A., \& Castro, A. (2011). Isótopos estables, fauna y artefactos en el estudio de los cazadores recolectores de la Costa Norte de Santa Cruz. Magallania, 39(1), 265-276.
Orquera, L., \& Piana, E. (2000). Composición de conchales de la costa del canal Beagle (Tierra del Fuego, República Argentina)-Primera Parte. Relaciones de la Sociedad Argentina de Antropología, XXV, 249-274.

Schiavini, A., Yorio , A., Gandini, P., Raya Rey, A., \& Dee Boersma, P. (2005). Los pingüinos de las costas argentinas: estado poblacional y conservación. Hornero, 20(1), 5-23.

Zilio, L. (2015). Prácticas mortuorias en la costa norte de Santa Cruz: arqueología de sociedades cazadoras recolectoras en paisajes costeros de la Patagonia argentina. Tesis doctoral. Facultad de Ciencias Naturales y Museo, Universidad Nacional de La Plata.

Zilio, L., Górdon, F., Béguelin, M., \& Castro, A. S. (2014). Paleodietas humanas en el sur del Golfo San Jorge (provincia de Santa Cruz) a partir del análisis de isótopos estables. Revista Argentina de Antropología Biológica, 16(1), 51-64. 